\title{
Fracture-risk calculators: Has their time come?
}

\author{
Mark J. Bolland MBChB PhD
}

See related research article by Langsetmo and colleagues, page E107

$\mathrm{O}$ steoporotic fractures occur in $30 \%-$ $50 \%$ of postmenopausal women and $10 \%-20 \%$ of older men, causing substantial morbidity, mortality and cost to society. Prevention of osteoporotic fractures is an important component of health care that relies on the accurate prediction of who is likely to sustain a fracture. Although predicting events is not easy, models that predict fracture have been developed and then validated in external populations. In this issue, Langsetmo and colleagues ${ }^{1}$ report the results of their validation study of one such model involving a Canadian cohort. We review the history of fracture prediction and discuss the use of fracture-risk calculators.

In 1994, a study group sponsored by the World Health Organization (WHO) recommended that postmenopausal women be grouped into four diagnostic categories of fracture risk (normal, osteopenia, osteoporosis and severe osteoporosis) based on bone mineral density $\mathrm{T}$ scores and history of fracture. ${ }^{2}$ Those recommendations were widely adopted and led to measurement of bone mineral density occupying a central place in strategies for fracture prevention. Nevertheless, numerous other risk factors for fracture exist (many of which are independent of bone mineral density), ${ }^{3}$ and most osteoporotic fractures occur among women who do not fulfill the WHO criteria for osteoporosis. ${ }^{4}$ For these reasons, models that integrate other risk factors for fracture in addition to bone mineral density might improve our predictive ability.

Predictive models have been reported for numerous conditions, but only a small proportion have entered clinical practice. Before clinical use, a model must be validated in populations different from those in which it was developed. Two key factors should be considered: discrimination and calibration. Discrimination is the ability of the model to distinguish between people with and without the outcome. It is assessed by the area under the receiver operating curve (or equivalent $\mathrm{C}$ statistic); a value of 1 indicates a perfect model, greater than 0.8 indicates excellent performance, 0.6 to 0.8 indicates moderate performance, less than 0.6 indicates weak performance, and 0.5 indicates performance no better than chance. ${ }^{5} \mathrm{Cal}-$ ibration is a measure of the goodness of fit of the model, and is assessed by comparing observed risk with predicted risk in a cohort divided by quintiles or deciles of predicted risk, and in subgroups defined by relevant clinical factors. ${ }^{5}$

Langsetmo and colleagues ${ }^{1}$ describe a carefully conducted validation study of an absolute risk calculator for fracture developed from the Dubbo Osteoporosis Epidemiology Study ${ }^{6}$ in Australia. The Dubbo calculator incorporates four clinical risk factors (age, sex, history of fractures and recent falls) with bone mineral density to produce estimated five- and ten-year risks of hip and osteoporotic fracture. The authors report that the Dubbo calculator has moderate predictive ability (greater for hip fracture than the broader category of osteoporotic fracture), is generally well-calibrated and is an improvement on the existing WHO recommendations $^{2}$ and, in men, the Canadian Association of Radiologists recommendations. ${ }^{10}$

A more widely known fracture-risk calculator is FRAX. A recent validation study of the country-specific FRAX-Canada tool involving the Manitoba Bone Density Program cohort showed similar findings, with good calibration and moderate predictive ability that was greater for hip fracture than major osteoporotic fracture. ${ }^{6}$

Absolute risk calculators for fracture have theoretical and practical advantages over earlier category-based systems. Fracture risk is expressed as a continuum, removing the somewhat arbitrary categorizations that otherwise occur. Clinically relevant reclassification of risk occurs, particularly in two settings: younger people with low bone mineral density but no clinical risk factors often have low risk of fracture over the short to medium term, whereas older people without osteoporosis by WHO criteria often have

\section{KEY POINTS}

- Absolute risk calculators improve fracture-risk prediction and should be used in clinical practice.

- Calculators should be validated in local cohorts before clinical use.

- Both validated in Canada, the Dubbo and FRAX-Canada calculators are suitable choices for assessing fracture risk in older Canadians. 
a high risk of fracture, especially if other clinical risk factors are present. Such reclassifications should alter prescribing practice. For these reasons, together with the improved performance in men, absolute fracture risk calculators are likely to supercede the current recommendations of the Canadian Association of Radiologists. ${ }^{10}$

The calculators have limitations. They have only moderate predictive ability, which is greater for hip fracture than osteoporotic fracture. However, their predictive ability is similar to that of other risk calculators, such as the Framingham cardiovascular risk calculator. ${ }^{8}$ Even moderately predictive calculators are useful in clinical practice and may outperform the risk assessments of clinicians. ${ }^{5}$ In addition, the calculators do not provide confidence intervals for the risk estimates, and they report estimates to one decimal place, creating the impression of greater precision than exists. Different country-specific FRAX tools can give markedly different estimates, highlighting the importance of local validation before use. ${ }^{9}$

Use of absolute risk for prediction of fracture will focus attention on treatment thresholds for fracture prevention. Using the Dubbo calculator, $90 \%$ of people older than age 80 years have an estimated ten-year fracture risk of greater than $20 \%,{ }^{1}$ which is defined by the Canadian Association of Radiologists as high risk. ${ }^{10}$ Similarly, the National Osteoporosis Foundation guidelines for osteoporosis management recommends pharmacologic treatment in $93 \%$ of

Table 1: Comparison of the Dubbo and FRAX fracture risk calculators

\begin{tabular}{|c|c|c|}
\hline Feature & Dubbo & FRAX \\
\hline \multicolumn{3}{|l|}{ Model development } \\
\hline Cohort(s) & $\begin{array}{l}\text { Single cohort in } \\
\text { Australia }\end{array}$ & $\begin{array}{l}20 \text { cohorts } \\
\text { worldwide }\end{array}$ \\
\hline Externally validated & Yes & Yes \\
\hline \multicolumn{3}{|c|}{ Model performance in Canadian cohort } \\
\hline \multicolumn{3}{|l|}{ Discrimination } \\
\hline Hip fracture & Excellent & Excellent \\
\hline Osteoporotic fracture & Moderate & Moderate \\
\hline Calibration & Good & Good \\
\hline \multicolumn{3}{|l|}{ Parameters of model } \\
\hline $\begin{array}{l}\text { Can be used without bone mineral } \\
\text { density values }\end{array}$ & Yes & Yes \\
\hline Number of variables & 5 & 11 \\
\hline \multicolumn{3}{|l|}{ Access to calculator } \\
\hline Simplified paper tables & Yes & Yes \\
\hline Web-based calculator & Yes & Yes \\
\hline Published equations or algorithm & Yes & No \\
\hline
\end{tabular}

women older than age 75 years. ${ }^{11}$ Despite classification as high-risk, most people in both of these situations will not sustain a fracture during the short to medium term. Calculating absolute risk reductions may help better inform patients of potential treatment benefits.

Keeping in mind their limitations, absolute risk calculators for fracture represent an important advance in fracture prediction and should be used in clinical practice. How should clinicians in Canada choose between the Dubbo and FRAX-Canada calculators? A comparison of the features of each is presented in Table 1. The performance of the calculators appears similar. Both calculators can be used without bone mineral density values, but their predictive ability drops noticeably. ${ }^{79}$ FRAX uses more variables than the Dubbo calculator, but the additional variables do not increase its predictive ability ${ }^{9,12}$ and add to the complexity and time needed to use the calculator. The equations of the Dubbo algorithm are freely available ${ }^{1}$ and can be incorporated into computer software, whereas the FRAX algorithm has not been published. Notwithstanding these differences, either calculator appears suitable for use in assessing fracture risk in older Canadians.

\section{References}

1. Langsetmo L, Nguyen TV, Nguyen ND, et al. Independent external validation of nomograms for predicting risk of lowtrauma fracture and hip-fracture. CMAJ 2011;183:E107-14.

2. Assessment of fracture risk and its application to screening postmenopausal osteoporosis. Technical Report series 843. Geneva: World Health Organization; 1994.

3. Kanis JA, Oden A, Johnell O, et al. The use of clinical risk factors enhances the performance of BMD in the prediction of hip and osteoporotic fractures in men and women. Osteoporos Int 2007; 18:1033-46.

4. Siris ES, Miller PD, Barrett-Connor E, et al. Identification and fracture outcomes of undiagnosed low bone mineral density in postmenopausal women: results from the National Osteoporosis Risk Assessment. JAMA 2001;286:2815-22.

5. Altman DG, Vergouwe $Y$, Royston $P$, et al. Prognosis and prognostic research: validating a prognostic model. BMJ 2009;338: b605.

6. Nguyen T, Sambrook P, Kelly P, et al. Prediction of osteoporotic fractures by postural instability and bone density. BMJ 1993 307:1111-5.

7. Leslie WD, Lix LM, Johansson H, et al. Independent clinical validation of a Canadian FRAX tool: fracture prediction and model calibration. J Bone Miner Res 2010;25:2350-8.

8. D'Agostino RB Sr, Grundy S, Sullivan LM, et al. Validation of the Framingham coronary heart disease prediction scores: results of a multiple ethnic groups investigation. JAMA 2001;286:180-7.

9. Bolland MJ, Siu AT, Mason BH et al. Evaluation of the FRAX and Garvan fracture risk calculators in older women. $J$ Bone Miner Res 2010 Aug. 18 [Epub ahead of print].

10. Siminoski K, Leslie WD, Frame H, et al. Recommendations for bone mineral density reporting in Canada. Can Assoc Radiol $J$ 2005;56:178-88

11. Donaldson MG, Cawthon PM, Lui LY, et al. Estimates of the proportion of older white women who would be recommended for pharmacologic treatment by the new U.S. National Osteoporosis Foundation Guidelines. J Bone Miner Res 2009;24:675-80.

12. Ensrud KE, Lui LY, Taylor BC, et al. A comparison of prediction models for fractures in older women: Is more better? Arch Intern Med 2009;169:2087-94.

Affiliations: Mark J. Bolland is with the Department of Medicine, University of Auckland, Auckland, New Zealand. 